\title{
Accessory spleen appearing as an intrasplenic pseudo-tumoral mass: A rare case report
}

\author{
YAN-FENG LÜ ${ }^{1}$, BING-BING HAN ${ }^{2}$, HUA-LONG YU ${ }^{1}$, ZHEN-HUA CUI ${ }^{1}$, ZHI-WEN LI $^{1}$ and JIAN-XIN WANG ${ }^{1}$ \\ ${ }^{1}$ Department of Anoproctology, The Second Hospital of Shandong University, Jinan, Shandong 250033; \\ ${ }^{2}$ Microcirculation Laboratory, Shangdong University of Traditional Chinese Medicine, Jinan, Shandong 250014, P.R. China
}

Received August 7, 2014; Accepted April 14, 2015

DOI: 10.3892/ol.2015.3331

\begin{abstract}
The current study presents a rare case of an accessory spleen that manifested as a solid intrasplenic pseudotumor. The affected patient was previously healthy. Upon examination with computed tomography (CT), an ovoid, soft-tissue mass of $4.1 \mathrm{~cm}$ in diameter was found on the upper pole of the spleen. Biochemical indices, such as blood routine and coagulation tests, and tumor marker analysis, revealed no abnormalities. Another CT scan was performed, but this failed to indicate whether the mass was benign or malignant. Therefore, the lesion was resected along with the spleen by laparoscopic surgery. The resected sample was subject to pathological examinations for final validation, and was finally diagnosed as an accessory spleen. The patient was followed up for six months with no signs of recurrence.
\end{abstract}

\section{Introduction}

An accessory spleen is a normal variant that is typically located at the splenic hilum, but is occasionally noted as a tumor-mimicking lesion located at multiple other sites, including the pelvis or gastrocolic ligament (1), and even the left scrotum (2) or mediastinum (3). Accessory spleens occur in $\sim 10 \%$ of the population and are usually located in the region of the splenic helum. Imaging tools are commonly adopted to make a diagnosis and surgical resection serves as the primary treatment (4). However, an accessory spleen manifesting as a solid intrasplenic pseudotumor has rarely been reported. Previous studies reported an accessory spleen presenting as an inflammatory mass and provided radiologic imaging of splenic abnormalities $(2,3,7)$. In the present study, the case of a male patient who was admitted to The Second

Correspondence to: Dr Jian-Xin Wang, Department of Anoproctology, The Second Hospital of Shandong University, 247 Beiyuan Street, Jinan, Shandong 250033, P.R. China

E-mail: jianxin211@sogou.com

Key words: accessory spleen, solid intrasplenic pseudotumor, diagnosis, treatment
Hospital of Shandong University (Jinan, China) presenting with a pseudotumoral accessory spleen is reported.

\section{Case report}

During the annual physical examination, a 38-year-old male presented with an occupied lesion in the spleen by screening ultrasonography in July 2011, and B-mode ultrasound detected no abnormality. In September 2011, the patient was admitted to The Second Hospital of Shandong University (Jinan. China) for further evaluation, and a computed tomography $(\mathrm{CT})$ scan showed an ovoid, soft-tissue density mass of $\sim 4.1 \mathrm{~cm}$ in diameter on the upper pole of the spleen (Fig. 1). A value of $41 \mathrm{HU}$ was recorded for the lesion on plain CT scan, while the portal phase CT value was $97 \mathrm{HU}$. The patient was diagnosed with a suspected hemangioma. The patient was previously healthy. Laboratory tests, including blood routine, coagulation, hepatic and renal function, $\alpha$-fetoprotein, carcinoembryonic antigen and cancer antigen 19-9 tests, following admission showed no abnormalities. The white blood cell count was $4.5 \times 10^{9} / 1$ and the hemoglobin level was $14 \mathrm{~g} / 1$. The lesion was resected together with the spleen by laparoscopic surgery. During the surgery, the lesion was found to be a $5 \times 4 \times 3-\mathrm{cm}$ tubercle with a smooth capsule, which protruded from the upper pole of the spleen, and the margin between it and the spleen was unclear. Upon examination, hematoxylin and eosin staining revealed slightly dilated splenic sinuses and clear margins between red and white pulps, which resembled normal splenic tissues, therefore an accessory spleen with partial fibrosis was diagnosed (Fig. 2). One day after surgery, the patient presented with fever and a body temperature of $38.2^{\circ} \mathrm{C}$, which was treated using sponge baths to cool the skin. Treatment was successful and the patient was discharged three days following surgery and was followed up for half a year at the Outpatient Department. Doppler ultrasound showed no sign of a thrombus. Platelet and coagulation functions were monitored regularly. At post-operative week two, the patient's platelet count was elevated to $900 \times 10^{9} / 1$, however, this returned to within the normal range $(\sim 100-$ $\left.300 \times 10^{9} / 1\right)$ after enteric-coated aspirin was prescribed and administered orally at a dose of $100 \mathrm{mg} / \mathrm{d}$ for 2 weeks, three times daily. At the last follow-up, in September 2014, the patient's platelet count was $180 \times 10^{9} / 1$ and the patient was asymptomatic. 

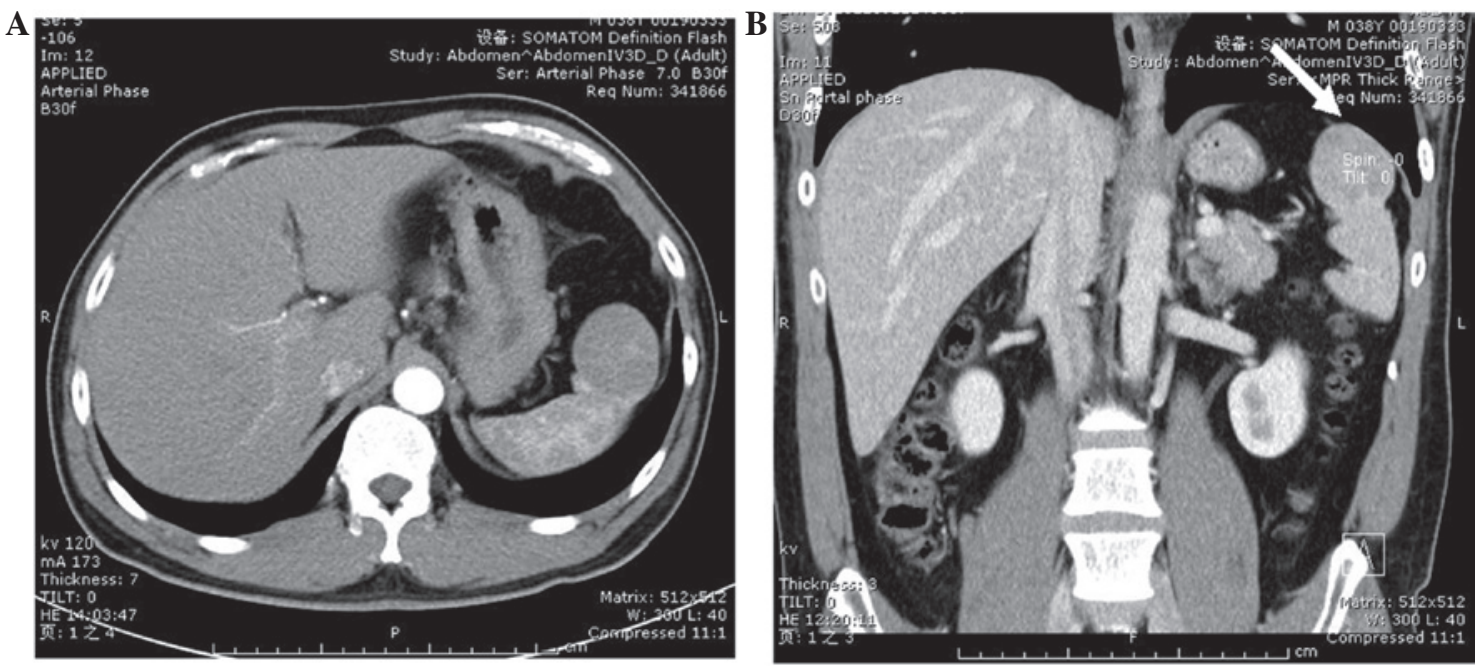

Figure 1. Computed tomography scans showing the accessory spleen located at the upper pole of the spleen (arrows). (A) Transverse plane at the arterial phase; (B) coronal plane at the portal phase.

A

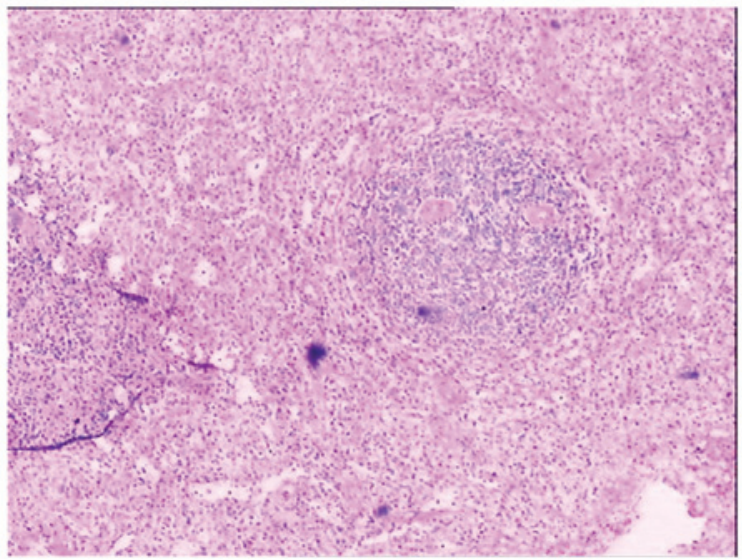

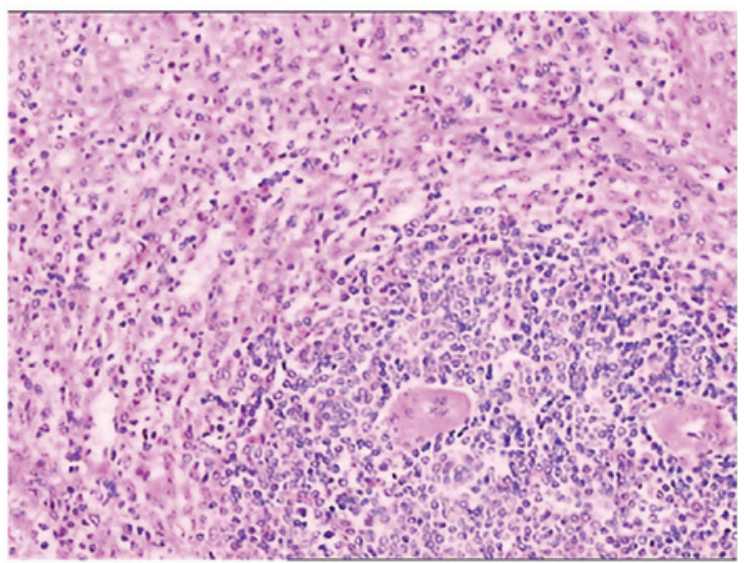

Figure 2. Pathological observation of the accessory spleen by hematoxylin and eosin staining showing (A) slightly dilated splenic sinuses and clear boundaries between the red and white pulps, resembling normal splenic tissues (magnification, x40); and revealing (B) medullary sinuses in the dilated medulla and splenic corpuscles in the white pulp within the marginal zone (magnification, $\mathrm{x} 200$ ).

\section{Discussion}

The accessory spleen is a type of congenital ectopic splenic tissue that shares a similar structure and function with the normal spleen. The accessory spleen can be completely separate from the proper spleen or attached by connective tissues. Under most circumstances, it has a different vascularity from that of the proper spleen. The accessory spleen may arise from the failure of fusion of the splenic anlage, located in the dorsal mesogastrium (5). The majority of accessory spleens are characterized as round or oval lesions, with a size ranging from a few millimeters to $>3 \mathrm{~cm}$ (6). Single or multiple accessory spleens may occur anywhere in the abdomen; they are one of the most common abnormalities with no clear syndromes or signs.

An accessory spleen can be readily diagnosed if it is located at a typical site. However, when located at atypical sites, accompanied by other lesions or manifested as corresponding symptoms, it may be confused with pancreatic (7) or left kidney tumors (8). In the current case, the lesion was located inside the proper spleen and presented with a poor boundary with surrounding normal splenic tissues. The lesion was large and solid, and the CT value of the mass increased during the enhanced phase. All these characteristics meant that is was more difficult to differentiate from a solid tumor.

An accessory spleen is incidentally found in $~ 10 \%$ of autopsy cases, but in 33\% of patients with hematological diseases (9), with a low incidence of pathological changes. Different clinical symptoms should be treated with differing methods. Prior to splenectomy, patients with hematological disorders should undergo imaging examinations to identify the presence, number and locations of any accessory spleens. During the splenectomy, all accessory spleens should be removed, or else they may enlarge and lead to the recurrence of the symptoms. For cases of splenic rupture, portal hypertension and benign splenic tumors, however, surgeons should pay attention to the presence of accessory spleens and keep them to preserve partial function of the spleen during the splenectomy. Theoretically, any pathological changes that can occur 
in the normal spleen can equally occur in the accessory spleen, including metastatic tumors. In the present case, blood routine and coagulation tests, and tumor marker analysis all showed no abnormalities, and imaging examinations revealed a neoplasm with an uncertain malignant or benign state. Therefore, surgical treatment was eventually considered. Post-operative pathological observations lead to the diagnosis of an accessory spleen, which emphasized the importance of thorough examinations pre-operatively and a fast pathological examination intraoperatively. CT scans may fail to identify accessory spleens that are too small or those located in the pelvis. More significantly, these lesions are difficult to differentiate from other entities when using CT scans alone (6). When an accessory spleen is suspected in a patient, Tc-99m-heat-denatured red blood cell (RBC) and Tc-99m-anti-D immunoglobulin G opsonized RBC scintigraphy should be undertaken to further confirm the diagnosis (10). The pathological and imaging characteristics of these two rare cases provide more clinical evidence for the clinical diagnosis and treatment of accessory spleen, which manifested as a solid pseudotumor.

\section{References}

1. Grinbaum R, Zamir O, Fields S and Hiller N: Torsion of an accessory spleen. Abdom Imaging 31: 110-112, 2006.
2. Seo T, Ito T, Watanabe $Y$ and Umeda T: Torsion of an accessory spleen presenting as an acute abdomen with an inflammatory mass. US, CT and MRI findings. Pediatr Radiol 24: 532-534, 1994.

3. Babcock TL, Coker DD, Haynes JL and Conklin HB: Infarction of an accessory spleen causing an acute abdomen. Am J Surg 127: 336-337, 1974.

4. Uchima S, Chijuwa K, Hiyoshi M, et al: Intrapancreatic accessory spleen mimicking endocrine tumor of the pancreas: Case report and review of the literature. J Gastrointest Surg 12: 1471-1473, 2008

5. Dodds WJ, Taylor AJ, Erickson SJ, Stewart ET and Lawson TL: Radiologic imaging of splenic anomalies. AJR Am J Roentgenol 155: 805-810, 1990.

6. Mortelé KJ, Mortelé B and Silverman SG: CT features of the accessory spleen. AJR Am J Roentgenol 183: 1653-1657, 2004.

7. Coote JM, Eyers PS, Walker A and Wells IP: Intra-abdominal bleeding caused by spontaneous rupture of an accessory spleen: the CT findings. Clin Radiol 54: 689-691, 1999.

8. Valls C, Monés L, Gumà A and López-Calonge E: Torsion of a wandering accessory spleen: CT findings. Abdom Imaging 23: 194-195, 1998.

9. Pérez Fontán FJ, Soler R, Santos M and Facio I: Accessory spleen torsion: US, CT and MR findings. Eur Radiol 11: 509-512, 2001.

10. Phom H, Kumar A, Tripathi M, Chandrashekar N, Choudhry VP, Malhotra A and Bal CS: Comparative evaluation of Tc-99m-heat-denatured RBC and Tc-99m-anti-D IgG opsonized RBC spleen planar and SPECT scintigraphy in the detection of accessory spleen in postsplenectomy patients with chronic idiopathic thrombocytopenic purpura. Clin Nucl Med 29: 403-409, 2004. 